\title{
๑BiblioCanto®
}

\section{REPOSITÓRIOS DIGITAIS UTILIZANDO WORDPRESS E MYSQL}

\section{DIGITAL REPOSITORIES USING WORDPRESS AND MYSQL}

\author{
Henry Poncio Cruz de Oliveira ${ }^{1}$ \\ henry.poncio@gmail.com \\ Ana Cláudia Cruz Córdula ${ }^{2}$ \\ anacordula@gmail.com \\ Nataniel José Amorin Fiuza ${ }^{3}$ \\ natan.fiuza@gmail.com \\ Mayane Paulino de Brito e Silva ${ }^{4}$ \\ mayanepaulino.b@gmail.com
}

Resumo: As demandas de organização, representação, armazenamento, acesso e uso de unidades organizacionais têm suscitado reflexões sobre o uso de softwares que possibilitem a construção de repositórios digitais. Os repositórios digitais são sistemas de informação que armazenam, preservam, divulgam e dão acesso à produção intelectual de comunidades científicas ou pessoas. São softwares utilizados para construção de repositórios: Eprints, Greeenstone, Nou-Rau, Fedora e DSpace. Este último largamente utilizado no Brasil. O presente trabalho objetiva apresentar um relato de experiência sobre

\footnotetext{
${ }^{1}$ Graduação em Física - UFPB. Mestre em Ciência da Informação - UFPB. Doutor em Ciência da Informação - UNESP. Docente do Departamento e do Programa de Pós-Graduação em Ciência da Informação e Coordenador do Portal de Periódicos Eletrônicos - UFPB. Lattes: http://lattes.cnpq.br/4231993792347599.

${ }^{2}$ Graduação em Fisioterapia - UNIPÊ. Graduação em Arquivologia - UFPB. Mestrado em Ciência da Informação UFPB. Doutorado em andamento em Ciência da Informação - UFPB. Docente do Departamento de Ciência da Informação - UFPB. Lattes: http://lattes.cnpq.br/9316211149718874

${ }^{3}$ Graduando do Curso de Arquivologia da UFPB. Lattes: http://lattes.cnpq.br/9770721645169680

${ }^{4}$ Graduação em Biblioteconomia - UFRN. Mestrado em andamento em Ciência da Informação UFPB. Lattes: http://lattes.cnpq.br/6621084090603012
} 


\section{Q Bibliocanto@}

a criação de repositórios digitais utilizando o Wordpress, uma ferramenta gratuita de CMS (Content Management System) escrita na linguagem PHP (Hypertext Preprocessor) com o banco de dados MySQL. A ferramenta Wordpress, com algumas customizações, pode ser utilizada para receber metadados de documentos digitais estruturados em textos, imagens, vídeos, animações, áudios, entre outros. Do ponto de vista metodológico, a presente pesquisa pode ser classificada como exploratória e descritiva. Utilizou-se uma abordagem analítica qualitativa para avaliar a customização e adaptação do Wordpress para o contexto dos repositórios digitais. O Wordpress, combinado com um banco de dados MySQL, apresentou funcionalidades e capacidade de customização adequadas para serem utilizados no contexto dos repositórios digitais, facilitando a inserção dos metadados e posterior recuperação dos documentos digitais.

Palavras-chave: Repositório digital. Wordpress. MySQL.

\section{INTRODUÇÃO}

É notável que a internet é um meio bastante utilizado para a disseminação da informação. Através dela é possível compartilhar as mais diversas informações em fração de segundos. Por outro lado, mesmo com toda a massa de informação que é criada a cada segundo e disponibilizada em proporções jamais vistas, é percebida a necessidade da criação de estratégias para a organização, representação e armazenamento da informação digital.

Neste contexto estão as demandas de organização, representação, armazenamento, acesso e uso de arquivos, que podem configurar-se como públicos ou privados, que tem suscitado reflexões sobre a construção de repositórios digitais. 


\section{Q Bibliocanto@}

Devido à crescente produção de informações digitais, aos compartilhamentos e aos inúmeros acessos que contribuem para construção do conhecimento e da memória, surge a necessidade da criação de repositórios digitais para armazenamento e disponibilização dessa informação digital.

Neste contexto existem vários softwares utilizados amplamente para construção de repositórios como o Eprints, Greeenstone, Nou-Rau, Fedora e DSpace. Estes softwares exigem um grande conhecimento técnico para implantação e utilização. $\mathrm{Na}$ contramão dos softwares utilizados para criação de repositórios, citamos o Wordpress, software com grande flexibilidade, capacidade de adaptação e personalização de sua aparência.

Nesta conjuntura, surgiu a ideia da utilização de um sistema de gerenciamento de conteúdo (CMS), o Wordpress, bastante utilizado atualmente na web, para gerir o processo de gestão de informações digitais. Trata-se de possibilitar o uso do Wordpress como ferramenta de repositório digital.

Buscando contribuir para o armazenamento, gestão e acesso da informação digital, o presente estudo tem por objetivo apresentar um relato de experiência sobre a criação de repositórios digitais utilizando o Wordpress, uma ferramenta gratuita de CMS (Content Management System) escrita na linguagem PHP (Hypertext Preprocessor) com o banco de dados MySQL.

Do ponto de vista metodológico, a presente pesquisa pode ser classificada como exploratória e descritiva. Utilizou-se uma abordagem analítica qualitativa para customizar e adaptar o Wordpress para o contexto dos repositórios digitais. Do ponto de vista procedimental, utilizamos o método de iteração para construir o referido repositório. 


\section{Q Bibliocanto@}

\section{INFORMAÇÃO E OS REPOSITÓRIOS DIGITAIS}

A informação digital tornou-se insumo fundamental para o desenvolvimento social, político e econômico das nações, bem como um fator proeminente para o desenvolvimento humano. $O$ estudioso português Fernando llharco, problematizando a informação tecnológica num plano filosófico, nos ajuda a compreender que a informação de natureza digital:

[...] surge no contexto da emergência de um novo tipo de informação, informação gerada, gerida, manipulada, armazenada, distribuída pela tecnologia. Ela surge como uma nova aproximação de topo ao homem e ao mundo, isto é, como um novo paradigma, no âmbito do qual se visa descrever e entender não apenas aquilo no qual o homem hoje está emerso, a informação tecnológica [informação digital], mas sob esse pretexto e no âmbito desse fenômeno de investigar e questionar os próprios fundamentos do que é, do que existe e do que somos nós, homens no mundo. (ILHARCO, 2003, p. 17).

Oliveira e Vidotti (2012, p. 275) acrescentam que toda informação digital é estruturada por meio da: "[...] linguagem binária e armazenada em suportes digitais, independente das características de volatilidade do suporte, cujo acesso e uso se dão através de equipamentos computacionais (hardware e software)".

O contexto de produção da informação digital tem demandado pesquisas científicas sobre as formas de armazenamento e as ferramentas adequadas para possibilitar o acesso e uso da informação.

Neste ínterim, estão os repositórios digitais que, segundo o Tesauro Brasileiro de Ciência da Informação (PINHEIRO; FERREZ, 2014, p. 195), são:

Mecanismos para administrar, armazenar e preservar conteúdos informacionais em formato eletrônico, e que podem ter como foco um assunto (repositórios temáticos) ou a 


\section{Q Bibliocanto@}

produção científica de uma instituição (repositórios institucionais). Muitos permitem o acesso universal e gratuito a seus conteúdos, que variam de acordo com a política de cada instituição. São coleções digitais de documentos de interesse para a pesquisa cientifica e, no caso dos institucionais, representam a sua memória cientifica.

Os Repositórios Digitais se configuram como canais de comunicação científica e que surgem a partir da necessidade de melhorar a disseminação da informação, reduzindo os custos antes empregados nos canais impressos (artigos, periódicos, dissertações, livros etc) (VILA, 2015).

Enfatiza-se que esses repositórios podem ser considerados uma inovação no gerenciamento da informação digital. Editoras, bibliotecas, arquivos e centros de informação em vários países estão criando grandes repositórios de informação digital, contendo diferentes tipos de conteúdos e formatos de arquivos digitais. (IBICT, 2016).

No Brasil, incentiva-se o depósito da publicação pelo pesquisador, ação denominada autoarquivamento, e a utilização de tecnologia aberta, permitindo o acesso gratuito em nível nacional e internacional.

A iniciativa de depósito da produção para acesso livre em repositórios institucionais seria:

a simples sugestão de que os pesquisadores depositem cópias de livre acesso de seus trabalhos aceitos em periódicos ou congressos, passando por diferentes tipos de incentivos para que seja feito o auto arquivamento até a obrigatoriedade institucional de o fazerem (SAYÃO, 2009, p. 18).

Traçando uma tipologia para os Repositórios Digitais, Leite (2009) os divide em: 


\section{Bibliocanto@}

a) Repositórios temáticos ou disciplinares: conjunto de trabalhos de uma área específica do conhecimento, particulariza a produção intelectual em função das áreas do conhecimento;

b) Repositório de teses e dissertações: lidam unicamente com teses e dissertações defendidas nos programas de pós-graduação das diversas áreas do conhecimento;

c) Repositório institucional: voltados à produção intelectual de uma instituição, especialmente universidades e institutos de pesquisa.

Para Sayão (2009, p. 10), os repositórios institucionais:

são entendidos hoje como elementos de uma rede ou infraestrutura informacional de um país ou de um domínio institucional destinados a garantir a guarda, preservação a longo prazo e, fundamentalmente, o livre acesso à produção científica de uma dada instituição.

Os repositórios têm finalidade de gerenciar a informação, como também, melhorar a comunicação interna e externa à instituição. Objetiva ainda maximizar a acessibilidade, o uso, a visibilidade e o impacto da produção científica institucional; retroalimentar a pesquisa científica e amparar os processos de ensino/aprendizagem; apoiar publicações científicas eletrônicas da instituição; contribuir na preservação e memória dos conteúdos digitais científicos ou acadêmicos produzidos pela instituição ou por seus membros; proporcionar input para a avaliação e monitoramento da produção científica. E ainda reunir, armazenar, organizar, recuperar e disseminar a produção da instituição (LEITE, 2009).

Alguns dos softwares livres usados são: DSpace, Eprints, Greeenstone, Nou-Rau e Fedora. Doravante, exporemos algumas de suas características, segundo Sayão (2009): 


\section{Bibliocanto@}

a) DSpace: Funcionalidade de armazenar, indexar, preservar e redistribuir documentos de pesquisa em formato digital. É um software livre, com download gratuito e versão em língua portuguesa. Fortemente indicado para Repositórios Institucionais, é focado em preservação digital a longo prazo;

b) Eprints: Possui maior e mais distribuída base instalada. Foi precursor no movimento de acesso livre.

c) Greenstone: Direcionado para criação e distribuição de coleções digitais, proporciona uma nova forma de organizar e publicar informações digitais na internet;

d) Nou-Rau: De código aberto, tem como objetivo implementar um sistema online para arquivamento, indexação, acesso controlado e busca de documentos digitais. Recebe documentos digitais em diversos formatos e os converte para texto puro;

e) Fedora: Infraestrutura ampla para o armazenamento, gestão e disseminação de objetos digitais complexos, incluindo o relacionamento entre eles. $\mathrm{O}$ conceito central do sistema é um poderoso modelo de objeto digital que estabelece como unidade de informação o "objeto digital Fedora".

\section{PROJETANDO REPOSITÓRIOS COM WORDPRESS}

Para Barcia (2008), os Content Management System (CMS), sistemas de gestão de conteúdos (SGC), são plataformas que integram ferramentas para criar e publicar conteúdos em tempo real, com interface intuitiva e dinâmica, sem a necessidade de conhecimentos específicos sobre programação.

Bax e Pereira (2002) definem CMS como sendo um sistema que permite a qualquer usuário da internet, mesmo àqueles com poucos conhecimentos informáticos, gerir um sítio dinâmico que separa a gestão de conteúdos do design gráfico das páginas. Os CMS possuem Frontend, para consulta de 


\section{Q Bibliocanto@}

conteúdos e Backend, restrita aos gestores da ferramenta.

O WordPress é um CMS disponibilizado em 2003 com poucas linhas de código para reduzir o esforço de escrita diária em ambientes digitais. Desde então tem crescido como ferramenta de blogging auto-hospedado, é usado em milhões de sites e visto por dezenas de milhões de pessoas todos os dias, mantido por uma grande comunidade de desenvolvedores e usuários. Tem evoluído para ser utilizado como sistema de gerenciamento de conteúdo completo e muito mais através dos milhares de plugins e widgets e temas. (ABOUT..., 2016).

Para a criação do exemplo de repositório digital foi necessário montar um ambiente virtual, no qual uma máquina desktop foi modificada para simular um servidor web com banco de dados MySQL, para isso foi utilizado o software ApacheFriends XAMPP versão $5.6 .15^{5}$. Este aplicativo instala todos os softwares necessários para a utilização do Wordpress, são eles: Apache 2.4.4 servidor web e o MySQL 5.5.32 banco de dados. O XAMPP tem uma instalação muito simples, após fazer o download do arquivo executável e seguir poucos passo e quase nenhuma configuração adicional.

Depois de todo o ambiente de simulação instalado foi feito download do Wordpress na versão $4.5 .2^{6}$, sua instalação necessita de alguns passos simples: descompactar o arquivo baixado em local correto para acesso pelo servidor web e criar uma novo banco de dados no MySQL.

Após descompactar e criar o novo banco dados, basta acessar pelo browser o diretório onde está descompactada a versão do Wordpress e seguir todos os passos exigidos pela instalação de forma intuitiva. No percurso de

\footnotetext{
${ }^{5}$ Disponível em <https://www.apachefriends.org/>. Acesso em: 10 abr. 2016.

${ }^{6}$ Disponível em <https://wordpress.org/download/>. Acesso em: 12 abr. 2016.
} 


\section{Bibliocantos}

instalação seguem as perguntas de configuração da página, da senha do banco de dados e também do nome do banco criado para o exemplo.

Com o Wordpress instalado foram feitas as adaptações de layout, necessárias para transformar a interface padrão de um blog em uma interface mais direcionada para um repositório digital, como remover opções de comentários em posts e exibição dos usuários autores das postagens, e os menus padrões de um blog.

Da instalação original, foram mantidas apenas as camadas: principal e sobre. Na barra lateral direita foram mantidos os itens: Busca, Categorias, Itens Recentes, Arquivos e Acesso tornando o layout simples e de fácil utilização.

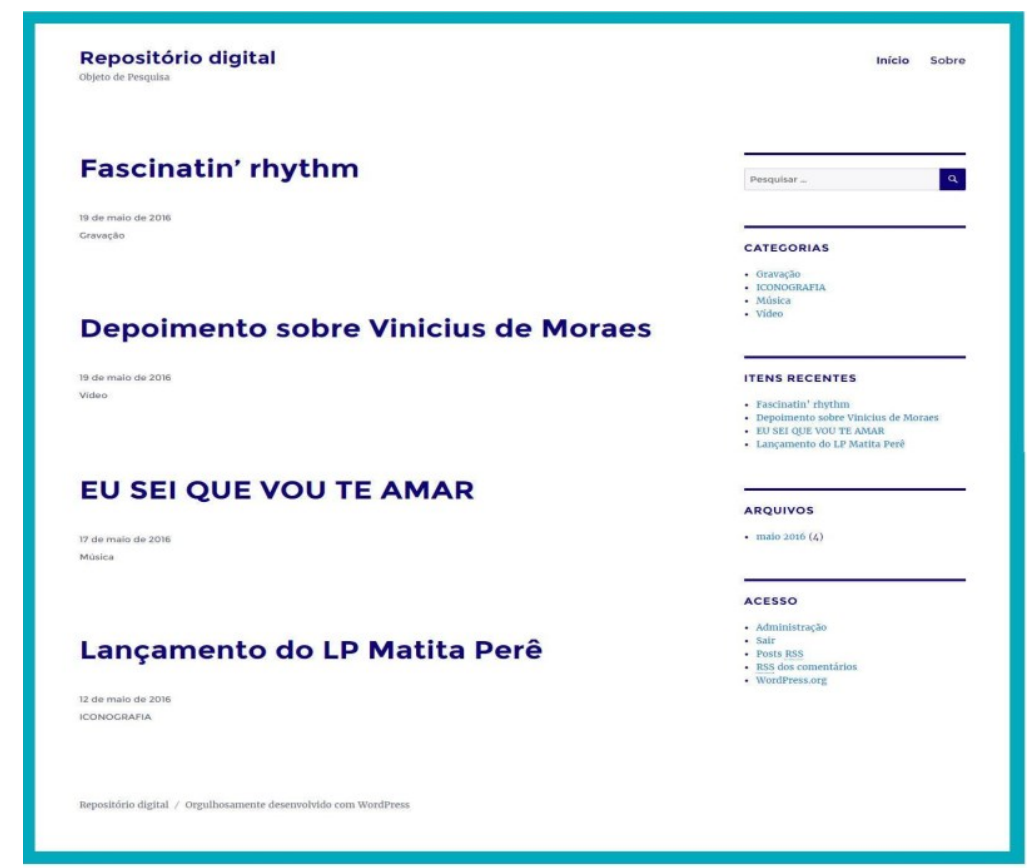

Figura 1 - Repositório construído utilizando Wordpress

Fonte: Dados da Pesquisa 


\section{Bibliocantos}

Para criar o cadastro dos documentos do repositório foi utilizado um plugin disponível no próprio Wordpress chamado Advanced Custom Fields (ACF) versão 4.4.7. Este plugin permite que seja alterada a tela de inclusão de um novo post. Por padrão a tela de inclusão de um post tem apenas dois campos título e texto. Para esta pesquisa foram adicionados outros campos, como também foi alterada a tela do formulário de inclusão, removendo os itens não necessários.

Com a utilização do ACF foram criados os seguintes campos no formulário de inclusão de um post:
a) Autor;
b) Palavras-chave;
c) Descrição;
d) Publicador;
e) Colaborador;
f) Data criação;
g) Tipo;
h) Formato;
i) Local;
j) Fonte;
k) Formato do arquivo;
l) Arquivo.

Vale destacar que os campos supracitados foram extraídos do padrão de metadados Dublin Core.

O campo "Tipo de arquivo" permite que o usuário armazene arquivos digitais a partir das seguintes possibilidades: Imagem, Vídeo, Som e Documento. Em seguida, aparece o botão que permite realizar o upload. 


\section{Bibliocantos}

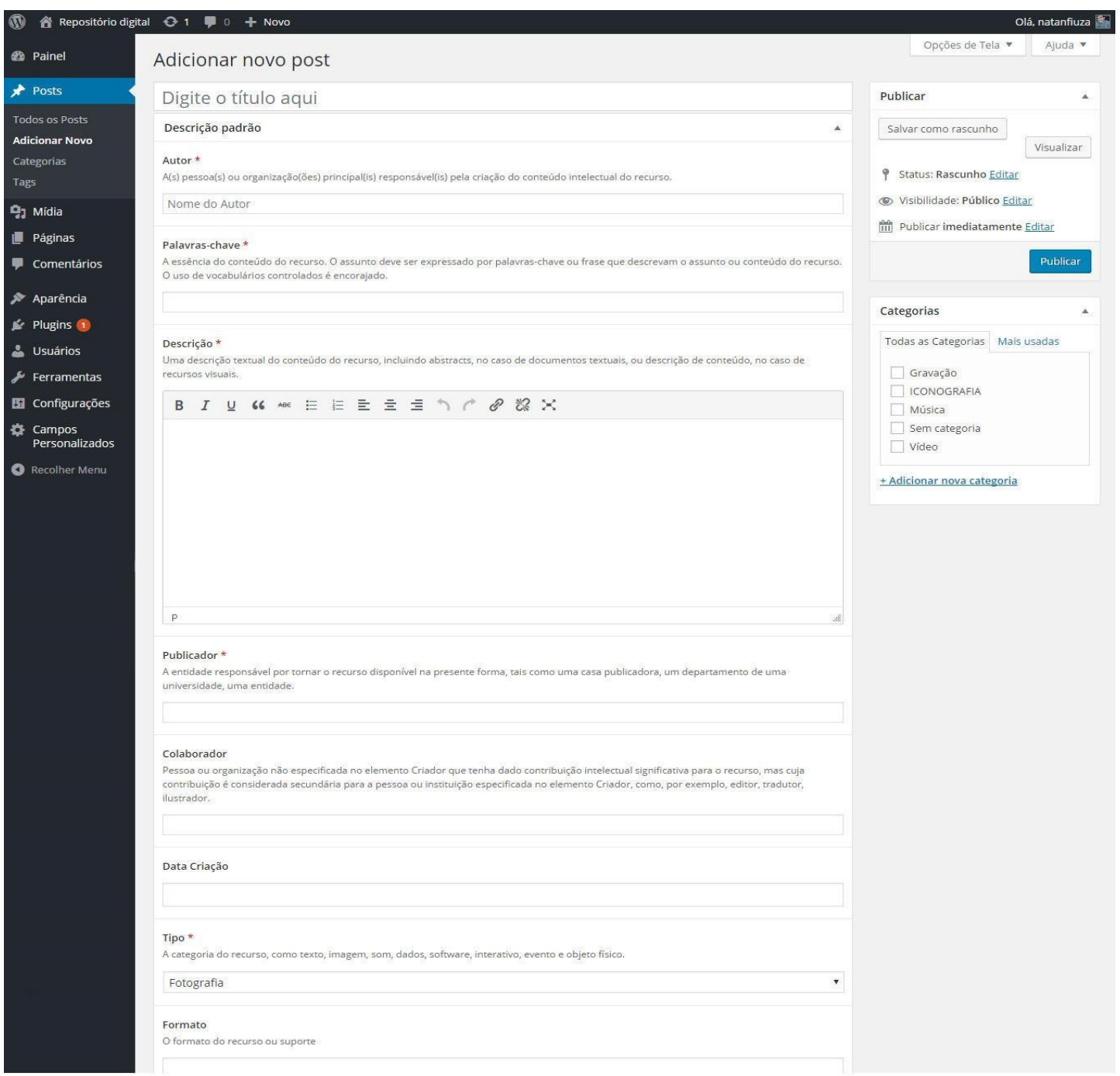

Figura 2 - Interface de Customização no Wordpress

Fonte: Dados da Pesquisa

A este ponto da customização foi necessária a alteração do código fonte do arquivo single.php do tema utilizado.

Esta foi a única alteração que exigiu conhecimentos de programação, pois o plugin ACF não disponibiliza a opção de edição na forma de exibição dos novos campos criados. 


\section{Bibliocantos}

\section{CONSIDERAÇÕES FINAIS}

O relato dos procedimentos necessários para fazer a customização de um repositório digital a partir de uma instalação padrão do Wordpress pode ajudar outros usuários na construção de repositórios em que necessite de um vasto conhecimento técnico.

O presente texto objetivou apresentar um relato de experiência relacionado ao uso do CMS Wordpress no contexto dos repositórios digitais. Do ponto de vista analítico, o Wordpress demonstrou ser uma ferramenta útil para criação de repositórios digitais.

Sobre a demanda de customização, foi necessário um conjunto pequeno de ações para customizar o CMS a fim de atender o armazenamento de documentos.

A ferramenta disponibiliza um sistema interno de busca que permite recuperar os documentos armazenados a partir dos atributos de metadados.

A principal dificuldade encontrada para a realização deste estudo foi a adaptação dos campos utilizando ferramentas de plugins e widgets existentes no próprio Wordpress. Mesmo assim houve pouca necessidade de intervenção em nível de programação direta no código fonte.

Com este estudo surge a necessidade de uma nova pesquisa para implementar um repositório digital confiável utilizando o modelo de referência Open Archival Information System (OAIS), além disso podem ser empreendidos estudos sobre Arquitetura da Informação, Usabilidade, Encontrabilidade, Acessibilidade, entre outros. 


\section{Q Bibliocanto@}

O Wordpress combinado com um banco de dados MySQL apresentou funcionalidades e capacidade de customização adequadas para serem utilizados no contexto dos repositórios digitais, facilitando a inserção dos metadados e posterior recuperação dos documentos digitais.

Abstract: The demands of organization, representation, storage, access and use of informational units has raised reflections on the use of software that allow the construction of digital repositories. Digital repositories are information systems that store, preserve, disseminate and provide access to the intellectual production of scientific or people communities. The software used for building repositories: Eprints, Greeenstone, Nou-Rau, Fedora and DSpace. The latter widely used in Brazil. This paper aims to present a research report on the creation of digital repositories using Wordpress, a free tool of CMS (Content Management System) written in PHP (Hypertext Preprocessor) with the MySQL database. The Wordpress tool with some customizations can be used to receive digital metadata documents structured text, images, videos, animations, audio, among others. From a methodological point of view, this research can be classified as exploratory and descriptive. We used a qualitative analytical approach to assess the customization and adaptation of Wordpress to the context of digital repositories. WordPress, combined with a MySQL database, presented features and customization capabilities suitable for use in the context of digital repositories, facilitating the integration of metadata and subsequent recovery of digital documents.

Keywords: Digital repositories. Wordpress. MySQL.

\section{REFERÊNCIAS}

ABOUT wordpress. Disponível em: <https://wordpress,org/about/>. Acesso em: 3 maio 2016. 


\section{Bibliocanto@}

BARCIA, L. M. G. A utilização da plataforma joomla! na escola. 2008. Dissertação (Mestrado em Ciências da Educação). Universidade Católica Portuguesa, 2008.

BAX, M. P.; PEREIRA, J. C. Introdução à Gestão de Conteúdos. 3o. Workshop Brasileiro de Inteligência Competitiva e Gestão do Conhecimento, 2002, São Paulo. Anais... 1o. Congresso Anual da Sociedade Brasileira de Gestão do Conhecimento.

INSTITUT BRASILEIRO DE INFORMAÇÃO EM CIÊNCIA E TECNOLOGIA. Repositórios Digitais. Disponível em: <http://www.ibict.br/informacao-paraciencia-tecnologia-e-inovacao\%20/repositorios-digitais>. Acesso em: 23 jul. 2016.

ILHARCO, Fernando. Filosofia da Informação: uma introdução à informação como fundação da acção, da comunicação e da decisão. Lisboa: Universidade Católica Editora, 2003.

LEITE, Fernando César Lima. Como gerenciar e ampliar a visibilidade da informação científica brasileira. Brasília: Ibict, 2009.

OLIVEIRA, Henry Poncio Cruz de; VIDOTTI, Silvana Aparecida Borsetti Gregório. Arquitetura da informação digital: conexões interdisciplinares dentro da abordagem sistêmica. In: CAVALCANTE, Lídia Eugênia; BENTES PINTO, Virgínia; VIDOTTI, Silvana Aparecida Borsetti Gregório. Ciência da informação e contemporaneidade: tessituras e olhares. Fortaleza: Edições UFC, 2012. p. 184-202.

PINHEIRO, Lena Vania Ribeiro; FERREZ, Helena Dodd. Tesauro Brasileiro de Ciência da Informação. Rio de Janeiro; Brasília: Instituto Brasileiro de Informação em Ciência e Tecnologia (lbict), 2014.

VILA, Monise Danielly Pessoa. As infovias do open access na Universidade Federal do Rio Grande do Norte. 2015. 90f. Trabalho de Conclusão de Curso (Graduação em Biblioteconomia) - Universidade Federal do Rio Grande do Norte, Natal, 2015.

SAYÃO, Luis et al. Implantação e gestão de repositórios institucionais: políticas, memória, livre acesso e preservação. Salvador: EDUFBA, 2009. 